\title{
La mutacion H63D del gen HFE se asocia con un riesgo aumentado de carcinoma hepatocelular
}

\author{
P. Ropero, O. Briceño, G. López Alonso¹, J. A. G. Agúndez², F. A. González Fernández, F. García Hoz \\ A. Villegas Martínez, M. Díaz-Rubio ${ }^{1}$ y J. M. Ladero ${ }^{1}$ \\ Servicios de Hematología y 'Aparato Digestivo. Hospital Clínico San Carlos. Universidad Complutense. Madrid. \\ ${ }^{2}$ Departamento de Farmacología. Universidad de Extremadura. Badajoz
}

\section{RESUMEN}

Objetivo: comprobar si las mutaciones del gen HFE, que pueden inducir sobrecarga hepática de hierro, guardan relación con el riesgo de desarrollar carcinoma hepatocelular $(\mathrm{CHC})$ en sujetos predispuestos a sufrir este tumor.

Material y métodos: se han incluido 196 pacientes (161 varones) diagnosticados de $\mathrm{CHC}$. Ninguno estaba diagnosticado de hemocromatosis. El grupo control estaba constituido por 181 sujetos sanos. Todos los sujetos eran españoles de raza blanca.

Las mutaciones C282Y y H63D del gen HFE se identificaron mediante reacción en cadena de polimerasa (PCR) sobre ADN genómico leucocitario utilizando enzimas de restricción específicas.

Resultados (casos/controles): 1. Distribución genotípica: a) mutación C282Y: 1/0 homocigotos, 12/23 heterocigotos, 183/158 normales ( $p=0,07$, n.s.); y b) mutación H63D: 9/5 homocigotos, 85/52 heterocigotos, 102/124 normales (odds ratio 2,00, IC95\% 1,29-3,12, p = 0,002). Cuatro casos y seis controles eran heterocigotos compuestos. 2. Frecuencias alélicas: a) mutación C282Y: normales 378/339, mutados 14/23 ( $\mathrm{p}=$ 0,11 , n.s.); b) mutación H63D: normales 289/300; mutados $103 / 62$ (odds ratio 1,72 , IC95\% $1,19-2,50, p=0,004$ ). No se observaron diferencias en relación con el sexo, la edad o la etiología (VHC, VHB, etílica o mixta) de la hepatopatía previa.

Conclusiones: la mutación C282Y no guarda relación con el riesgo de desarrollar $\mathrm{CHC}$ en sujetos sin hemocromatosis conocida. La posesión de la mutación H63D se asocia con un riesgo aumentado de desarrollar $\mathrm{CHC}$ independientemente de la etiología de la hepatopatía crónica subyacente.

Palabras clave: Gen HFE. C282Y. H63D. Carcinoma hepatocelular. Etanol. Virus de hepatitis B. Virus de hepatitis C.

\begin{abstract}
Aim: to disclose whether mutations in the HFE gene inducing liver iron overload are related to the risk of hepatocellular carcinoma (HCC) in otherwise predisposed patients.

Patients and methods: one hundred and ninety-six patients (161 males) diagnosed with HCC and 181 healthy controls were included in the study. All subjects were white Spaniards.

C282Y and H63D mutations in the HFE gene were identified in leucocyte genomic DNA using a polymerase chain reaction (PCR) and specific restriction enzymes.

Results (cases/controls): 1. Genotype distribution: a) C282Y mutation: homozygotes $1 / 0$, heterozygotes $12 / 23$, wild type 183/158 ( $p=0.07$, non significant); b) H63D mutation: homozygotes $9 / 5$, heterozygotes 85/52, wild type 102/124 (0dds ratio $2.00,95 \%$ C.I. $1.29-3.12, p=0.002$. Four cases and 6 controls were carriers of heterozygous mixed genotypes. 2. Allele frequencies: a) C282Y mutation: wild type allele 378/339, mutated allele $14 / 23$ ( $p=0.11$, non significant); b) H63D mutation: wild type allele 289/300, mutated allele 103/62 (0dds ratio 1.72, 95\% C.I. 1.19-2.50, p = 0.004). Age at diagnosis, gender and etiology of the underlying liver disease do not influence these findings.

Conclusion: the C282Y mutation in the HFE gene is not related to the risk of HCC in non-hemochromatosis patients. The H63D mutation is associated with a higher risk of HCC in cirrhotic patients irrespective of their underlying liver disease.
\end{abstract}

Key words: HFE gene. C282Y mutation. H63D mutation. Hepatocellular carcinoma. Hepatitis B. Hepatitis C. Ethanol abuse.

Ropero P, Briceño O, López Alonso G, Agúndez JAG, González Fernández FA, García Hoz F, Villegas Martínez A, Díaz-Rubio M, Ladero JM. La mutación H63D del gen HFE se asocia con un riesgo aumentado de carcinoma hepatocelular. Rev Esp Enferm Dig 2007; 99: 376-381.

Recibido: 15-12-06

Aceptado: 27-02-07.

Correspondencia: José M. Ladero. C/ Modesto Lafuente, 46, 5C. 28003 Madrid.e-mail: jladero.hcsc@salud.madrid.org

\section{INTRODUCCIÓN}

El carcinoma hepatocelular (CHC) es el cuarto cáncer más frecuente en el mundo, con una incidencia de 3 a 80 casos por 100.000 habitantes/año (1) que está en aumen- 
to (2), aunque con grandes variaciones geográficas (3). Ocupa el tercer lugar en las muertes por cáncer (4). Más del $80 \%$ de los casos aparecen sobre hígados cirróticos (5) proporción que en España se eleva al 90\% (6). El riesgo varía con la etiología de la cirrosis y es máximo en la secundaria a hemocromatosis $(7,8)$, aunque probablemente menor de lo que se pensaba anteriormente (9). En Europa el factor de riesgo más importante es la cirrosis por el virus de la hepatitis C (VHC), solo o asociado a abuso de etanol $(10,11)$. En nuestro centro, el $68 \%$ de los CHC asientan sobre cirrosis por $\mathrm{VHC}$ (12).

La identificación por Feder y cols. (13) del gen de la hemocromatosis (HFE) y de la mutación Cys282Tyr ha permitido establecer la base genética de la mayoría de los casos de hemocromatosis hereditaria en sujetos de raza blanca de estirpe anglo-céltica (14), aunque en los países europeos de la cuenca mediterránea la prevalencia de la homocigosidad para la mutación $\mathrm{C} 282 \mathrm{Y}$ en pacientes con hemocromatosis genética es sólo del 64\% (15). Además, sólo el $50 \%$ de los homocigotos para la mutación C282Y desarrollan fenotípicamente hemocromatosis y otro $25 \%$ tienen sobrecarga de hierro sin repercusión clínica (16), por lo que deben de existir otros factores que modulen la patogenicidad de esta mutación. Uno de ellos es una segunda mutación del gen $H F E$, denominada H63D, cuya coincidencia en heterocigosis mixta con la mutación C282Y puede inducir un síndrome clínico indiferenciable del que desarrollan los homocigotos C282Y (17).

Las mutaciones del gen $H F E$ pueden ser un cofactor que agrave otras enfermedades crónicas del hígado (18). Existen estudios sobre la posible relación de las mutaciones del gen $H F E$ con el riesgo y la evolución de diferentes enfermedades hepáticas, como la infección crónica por VHC (19-22), la hepatopatía alcohólica $(23,24)$, la porfiria cutánea tarda (25) o la esteatohepatitis no alcohólica (26-28), con resultados controvertidos. La justificación que se esgrime para la práctica de estos estudios se basa en la presunta sobrecarga férrica inducida por la posesión de alguna mutación del gen HFE, capaz de inducir daño hepático al aumentar el estrés oxidativo (29). En el caso específico del CHC, que es el objeto de este estudio, la sobrecarga hepática de hierro podría ser carcinógena a través del estímulo directo de la proliferación celular (30) y de la inducción de daño en el ADN, especialmente de mutaciones en el gen p53 $(31,32)$, pero además, de forma indirecta, por el ya citado incremento del estrés oxidativo que induciría peroxidación de los lípidos de las membranas, daño del ADN por formación de adducts y estímulo de la células estrelladas con la consiguiente aceleración de la fibrogénesis $(29,33)$.

Está demostrado que los genotipos C282Y/C282Y y C282Y/H63D se asocian generalmente a un cierto grado de sobrecarga férrica, aunque con menor frecuencia a un fenotipo hemocromatósico $(16,17)$. Un reciente estudio sobre una amplia casuística ha comprobado que la posesión de cualquier mutación del gen $H F E$ incrementa los índices de hierro hepático en pacientes con infección cró- nica por el VHC (34), lo que refrenda los hallazgos comunicados previamente sobre series más cortas o basados únicamente en determinaciones de parámetros férricos en sangre (35-41). Sin embargo, la repercusión de la mutación H63D, tanto en homo- como en heterocigosis, sobre el depósito de hierro en el organismo es controvertida y diversos grupos no han encontrado asociación entre esta mutación y el contenido hepático de hierro (4245). Es destacable que la mutación H67D del ratón, equivalente a la H63D humana, favorece el acúmulo hepático de hierro (46).

El gen $H F E$ codifica la síntesis de una molécula de clase I del complejo mayor de histocompatibilidad (MHC), cuyo surco de fijación de péptidos es demasiado estrecho para permitir la presentación de antígenos (47), pero las mutaciones del gen guardan relación con alteraciones funcionales de las moléculas clásicas de clase I del $\mathrm{MHC}$, lo que indica que el gen $H F E$ puede intervenir en la respuesta inmune (48), que es un elemento protagonista en la evolución de las hepatopatías crónicas sobre las que se insertan la mayor parte de los casos de CHC.

Este estudio se ha realizado para investigar la posible relación entre las mutaciones C282Y y H63D del gen $H F E$ y el riesgo de desarrollar CHC en pacientes españoles diagnosticados de este tumor y su posible asociación con otros factores etiológicos.

\section{MATERIAL Y MÉTODOS}

Se han incluido 196 enfermos consecutivos (161 varones, edad media 66,1 años, D.E. 9,7, intervalo 29-88), de raza blanca y nacionalidad española, diagnosticados de CHC en el Servicio de Aparato Digestivo del Hospital Clínico San Carlos desde enero de 1994 hasta septiembre de 2003. El diagnóstico de CHC se basó en los resultados de la anatomía patológica o en una concentración de alfa fetoproteína > $399 \mathrm{ng} / \mathrm{dl}$ en presencia de una masa hepática identificada en un estudio de imagen. La tabla I muestra las características demográficas y epidemiológicas de los pacientes. Todos los enfermos dieron su consentimiento para la obtención de la muestra sanguínea tras ser debidamente informados sobre la naturaleza del estudio.

El grupo control está constituido por 181 sujetos presuntamente sanos, donantes de sangre, que autorizaron la toma de la muestra sanguínea. Sólo se eliminaron aquellos cuya donación fuera descartada por cualquier motivo y los que guardaran parentesco con otro sujeto control. La reserva sobre los datos de estos sujetos es absoluta, por lo que no se conoce su sexo ni su edad, aunque no se seleccionaron muestras de personas de raza no blanca o nacionalidad no española.

El ADN se obtuvo de leucocitos de sangre periférica mediante un procedimiento de extracción estándar (49). El análisis del gen HFE se llevó a cabo mediante PCR (reacción en cadena de la polimerasa) del ADN genómico, amplificando la región del gen portadora de cada mu- 
Tabla I. Características de los 196 pacientes

Sexo (varones/mujeres): 161/35

Edad media: 66,1 años, DE 9,7 (intervalo: 29-88)

Diagnóstico:

Masa(s) hepática(s) y al menos uno de los siguientes:

Confirmación anatomopatológica: 122 casos

Alfa-feto proteína > $399 \mathrm{ng} / \mathrm{dl}$ : 113 casos

Ambos criterios: 41 casos

Consumo de etanol excesivo (> $50 \mathrm{~g} /$ día): 93 casos (91 varones).

HbsAg positivo/negativo/desconocido: 12/171/13*

Anti HBC positivo/negativo/desconocido: 71/114/11**

Anti VHC positivo/negativo/desconocido: 128/61/7***

*: En estos 13 casos anti-HBc negativo en 2 casos y desconocido en 11 casos; VHC positivo en 6 casos, negativo en 3 y desconocido en $4 ; * *$ : 6 de estos 11 casos tenían anti-VHC $+; * * *$ : 2 de estos 7 casos tenían algún marcador de VHB positivo. Quince sujetos eran $\mathrm{HbsAg}$, antiHBc y anti-VHC negativos y no habían consumido alcohol.

tación, empleando para ello oligonucleótidos previamente descritos $(13,50)$.

El producto amplificado, portador del codón 282, fue un fragmento de 387 pares de bases (pb), el cual fue digerido con la enzima de restricción Rsa I con una diana GT $\downarrow$ AC localizada en el alelo mutado. En el caso de la mutación H63D, el fragmento amplificado de 208 pb fue digerido con la enzima de restricción Dpn II, cuya diana ( $\downarrow$ GATC) está localizada en el alelo normal.

El análisis estadístico se realizó aplicando tests para variables continuas independientes (test $t$ de Student o U de Mann-Whitney, según procediera), y el criterio Chi cuadrado (Mantel-Haenszel) para variables categóricas, calculando el valor de odds ratio y el intervalo de confianza al $95 \%$ en variables dicotómicas, aplicando además la Chi al cuadrado de tendencia lineal cuando era pertinente (valoración de efecto dosis-gen) y la corrección de Bonferroni en comparaciones múltiples. La posible influencia de otros factores de riesgo (edad, sexo, etiología) se analizó mediante tablas de contingencia, ya que al no haber un grupo control equiparable para estas variables, no es posible realizar un análisis multivariante. La hipótesis nula se descartó cuando $\mathrm{p}<0,05$ o cuando el intervalo de confianza no incluía la unidad. Se emplearon los paquetes informáticos EpiInfo 2000, de los Center for Disease Control \& Prevention (Atlanta) y SPSS 11.5.

\section{RESULTADOS}

La distribución de los genotipos HFE se muestra en la tabla II, y la frecuencia alélica de las mutaciones en la tabla III. Un paciente, que no estaba diagnosticado de hemocromatosis, era homocigoto para la mutación C282Y. La frecuencia alélica y la proporción de heterocigotos simples C282Y eran bajas en ambos grupos y no muestran diferencias significativas. Cuatro pacientes y seis controles eran heterocigotos mixtos C282Y/H63D. De los 13 casos portadores de la mutación C282Y, cuatro eran anti-VHC positivos, tres bebedores excesivos de etanol y en seis coincidían ambos factores.

En el grupo de casos hay un exceso significativo de portadores de la mutación H63D (48,0 vs. 31,5\%) (Tabla II) y de la frecuencia alélica de esta mutación $(0,263 \mathrm{vs}$. 0,171 ) (Tabla III). Esta diferencia se mantiene al eliminar del análisis a los 13 casos y 23 controles portadores de la mutación C282Y, apreciándose un posible efecto dosisgen mediante el test de tendencia lineal (Tabla II, columna derecha).

Tabla II. Distribución de los genotipos HFE en casos y controles

\begin{tabular}{|c|c|c|c|c|c|c|c|}
\hline $\begin{array}{l}\text { Mutación } \\
\text { HFE }\end{array}$ & Grupo & $\begin{array}{l}\operatorname{Sin} \\
\text { mutaciones } \\
(\%)\end{array}$ & $\begin{array}{c}\text { Portadores } \\
\text { Homocigotos/heterocigotos } \\
\text { compuestos/heterocigotos } \\
\text { simples }\end{array}$ & Total serie & Estadística & $\begin{array}{l}\text { jietos sin C282 } \\
\text { dio mutación H }\end{array}$ & $\begin{array}{l}Y \\
\text { (63D) }\end{array}$ \\
\hline C282Y & $\begin{array}{c}\text { Casos } \\
\text { Controles }\end{array}$ & $\begin{array}{l}183(93,4) \\
158(87,3)\end{array}$ & $\begin{array}{c}13(1 / 4 / 8) \\
23(0 / 6 / 17)\end{array}$ & $\begin{array}{c}\text { Odds ratio }=0,49 \\
\text { IC } 95 \%=0,23-1,05 \\
C h i^{2}=3,35 \\
p=0,07\end{array}$ & $\begin{array}{l}\text { Genotipo H63D } \\
\text { Wild type } \\
\text { Heterocigoto } \\
\text { Homocigoto }\end{array}$ & $\begin{array}{c}\text { Casos (183) } \\
93 \\
81 \\
9\end{array}$ & $\begin{array}{c}\text { Controles (158) } \\
107 \\
46 \\
5\end{array}$ \\
\hline$H 63 D$ & $\begin{array}{c}\text { Casos } \\
\text { Controles }\end{array}$ & $\begin{array}{c}102(52) \\
124(68,5)\end{array}$ & $\begin{array}{l}94(9 / 4 / 81) \\
57(5 / 6 / 46)\end{array}$ & $\begin{array}{c}\text { Odds ratio }=2 \\
\text { IC } 95 \%=1,29-3,12 \\
C^{2} i^{2}=9,95 \\
p=0,002\end{array}$ & $\begin{array}{l}\text { Odds ratio (porta } \\
\text { IC95\% }=1,25-3, \\
\mathrm{Chi}^{2}=8,63, p= \\
\mathrm{Chi}^{2} \text { de tendencia }\end{array}$ & $\begin{array}{l}\text { dores vs. wild-t } \\
28 \\
, 004 \\
\text { lineal }=8,91 ;\end{array}$ & $\begin{array}{l}\text { ype) }=2,03 \\
p=0,003\end{array}$ \\
\hline
\end{tabular}

Tabla III. Frecuencia alélica en casos y controles

\begin{tabular}{|c|c|c|c|c|c|c|c|}
\hline \multirow[t]{2}{*}{ Alelo HFE } & \multirow[t]{2}{*}{ Grupo } & \multirow{2}{*}{$\begin{array}{l}\text { Normal } \\
\text { (frecuencia) }\end{array}$} & \multirow{2}{*}{$\begin{array}{c}\text { Mutado } \\
\text { (frecuencia) }\end{array}$} & \multicolumn{4}{|c|}{ Estadistica } \\
\hline & & & & Odds ratio & IC95\% & $C h i^{2}$ & $p$ \\
\hline \multirow[t]{2}{*}{ C282Y } & Casos & $378(0,964)$ & $14(0,036)$ & 0,55 & $0,26-1,13$ & 2,55 & 0,11 \\
\hline & Controles & $339(0,937)$ & $23(0,063)$ & & & & \\
\hline H63D & $\begin{array}{l}\text { Casos } \\
\text { Controles }\end{array}$ & $\begin{array}{c}289(0,737) \\
300(0,83)\end{array}$ & $\begin{array}{c}103(0,263) \\
62(0,17)\end{array}$ & 1,72 & $1,19-2,50$ & 8,69 & 0,004 \\
\hline
\end{tabular}


Doce enfermos (11 varones) eran HbsAg positivos. Ninguno de ellos era portador de la mutación C282Y, pero 10 eran portadores de la mutación H63D (9 heterocigotos y 1 homocigoto). Este exceso alcanza significación estadística (Chi al cuadrado 6,597, p = 0,01). En 6 de estos 12 pacientes coincidían otros factores de riesgo (infección por VHC en 2 casos, abuso de etanol en 2 casos, ambos en 2 casos).

No se encontraron variaciones significativas en la frecuencia de las mutaciones del gen $H F E$ en relación con el sexo, la edad al diagnóstico, la positividad o negatividad para antiVHC y antiHBc y el consumo excesivo de etanol.

\section{DISCUSIÓN}

En este estudio hemos detectado un exceso de portadores de la mutación H63D del gen $H F E$ en pacientes con $\mathrm{CHC}$ en comparación con un grupo de controles sanos de la misma nacionalidad y origen étnico, reclutados en la ciudad de Madrid. La prevalencia de la mutación H63D en el grupo control (frecuencia alélica 0,171 ) es ligeramente inferior a la detectada en otro grupo de controles de la misma nacionalidad y raza, aunque de una zona geográfica diferente (Cataluña); los autores de este estudio (51) señalan que en sus controles se detecta una de las frecuencias más elevadas del alelo H63D comunicadas en la literatura. En un estudio realizado por nuestro grupo en sangre de cordón umbilical de 298 neonatos hijos de españoles de raza blanca nacidos en Madrid, las frecuencias alélicas fueron respectivamente de 0,018 para la mutación C282Y y de 0,191 para la mutación H63D (52). Si hubiéramos utilizado este grupo como control, se mantendría la significación estadística de las diferencias detectadas en los porcentajes de portadores de la mutación H63D $(p=0,006)$ y en la frecuencia alélica de esta mutación ( $\mathrm{p}=0,017)$. Además, estos datos son muy similares a los detectados por otros grupos en el área mediterránea europea occidental (53).

La mutación C282Y del gen $H F E$ está presente en 13 casos y 23 controles, con una frecuencia alélica de 0,036 y 0,063 , respectivamente (Tabla III). Estos datos confirman la baja prevalencia de esta mutación en la población española ya señalada por otros autores (51). Aunque esta diferencia no es significativa, apunta en sentido contrario al que cabría esperar si la posible relación de riesgo de esta mutación con el CHC se debiera a que induce sobrecarga de hierro (54), algo indudable cuando aparece en homocigosis (51) pero también comprobado, aunque en grado mucho menor, cuando está en heterocigosis (34). No hemos podido valorar el contenido hepático de hierro de nuestros pacientes, ya que la mayor parte de los diagnósticos histológicos se alcanzaron mediante estudio de citología obtenida con punción aspirativa con aguja fina y la ferritinemia no refleja el contenido corporal de hierro en pacientes cirróticos con CHC. En un estu- dio en 35 pacientes con $\mathrm{CHC}$ desarrollado sobre hígado no cirrótico se detectó sobrecarga de hierro en el parénquima hepático en 19 de ellos, de los cuales 7 eran portadores $\mathrm{C} 282 \mathrm{Y}$, mientras que los 16 pacientes sin sobrecarga férrica no tenían ninguna mutación del gen HFE (55). En la revisión bibliográfica efectuada algunos trabajos detectan un exceso de mutación C282Y; especial interés tiene el estudio de Lauret y cols. (56) realizado en España, que detectaron un exceso significativo de heterocigotos $\mathrm{C} 282 \mathrm{Y}$ en 43 pacientes con $\mathrm{CHC}$ sobre cirrosis alcohólica $(20,9 \%)$ en comparación con 136 sujetos con cirrosis alcohólica sin tumor añadido $(4,4 \%)$, pero no en 34 pacientes con $\mathrm{CHC}$ sobre cirrosis por VHC. Hallerbrand y cols. (57), en un grupo de 137 enfermos con CHC, no identificaron ningún homocigoto C282Y, pero detectaron una proporción significativamente mayor de heterocigotos $(12,4 \%)$ en comparación con enfermos cirróticos sin $\mathrm{CHC}(3,7 \%, \mathrm{p}=0,020)$ y con sujetos sanos $(4,8 \%, p=0,031)$. Fargion y cols. (58) detectaron un exceso de la mutación $\mathrm{C} 282 \mathrm{Y}$ en 81 pacientes con $\mathrm{CHC}$ frente a controles $(8,6 v s .1,6 \%, \mathrm{p}<0,03)$, sugiriendo que la posesión de esta mutación puede incrementar el riesgo inherente a la cirrosis etílica o por VHC, pero la frecuencia alélica de la mutación en sus controles es más baja que en otra serie (53) estudiada en el mismo país $(3,2 \%)$. Francazani y cols. (59) y Shi y cols. (60) encontraron una inequívoca relación entre el riesgo de padecer hepatoma y el estado de portador de la mutación C282Y sólo en aquellos enfermos con hepatitis crónica B y de sexo masculino. Otros estudios confirman la relación de la mutación C282Y con el riesgo de CHC sólo cuando está en homocigosis $(61,62)$, u ofrecen resultados negativos (6365). Beckman y cols. (66) han identificado una relación significativa de riesgo cuando coinciden la mutación C282Y del gen $H F E$ y el genotipo Ser/Ser en posición 142 del gen del receptor de transferrina, aunque no si aparecen por separado. Aunque estos autores no comentan el hecho, destaca que el $22 \%$ de estos pacientes eran portadores de la mutación C282Y.

En este estudio hemos detectado un exceso significativo de portadores la mutación H63D que no había sido señalado antes en la literatura. Esta diferencia es máxima en portadores de $\mathrm{HbsAg}$, pero afecta a todos los grupos etiológicos y es independiente de variables como la edad y el sexo. Tan sólo dos estudios $(64,66)$ han detectado un exceso cuantitativamente menor y que no alcanza significación estadística debido al pequeño tamaño muestral.

Es difícil relacionar el riesgo asociado con la posesión de la mutación H63D con una hipotética sobrecarga férrica, que si bien puede existir, como se ha señalado anteriormente, es mucho menor que la que puede asociarse a la mutación C282Y. Una explicación alternativa se basaría en la adscripción del gen $H F E$ a las moléculas de clase I del complejo mayor de histocompatibilidad y en el posible desequilibrio de ligamiento entre la mutación H63D y el haplotipo HLA-A29-B44 (48), pero por el momento la posible interacción entre las mutaciones del gen 
$H F E$ y la función inmune sólo se ha empezado a analizar en lo tocante a la mutación C282Y (67).

Puede conjeturarse que las mutaciones del gen $H F E$ facilitan la progresión hacia la cirrosis que precede habitualmente a la aparición de CHC, en lugar o además de ejercer un efecto oncogénico directo. En un estudio previo no hemos detectado diferencias en la gravedad histológica de la hepatitis crónica $\mathrm{C}$ relacionadas con el genotipo HFE (22), aunque Geier y cols. (68) sí encontraron una mayor afectación en los enfermos portadores de cualquiera de las dos mutaciones. En lo relativo a la hepatopatía alcohólica, hemos detectado una mayor prevalencia de la mutación H63D en sujetos con cirrosis de esta etiología con respecto a la población general (23).

Nuestro estudio, basado en una amplia casuística, sugiere que la posesión de la mutación H63D es un factor de riesgo para el desarrollo de $\mathrm{CHC}$ en sujetos con factores predisponentes, especialmente cirrosis hepática debida a infección crónica por VHC y/o a abuso de etanol e infección crónica por VHB. Se necesitan más estudios sobre las funciones del gen $H F E$ y las repercusiones que la mutación H63D puede tener sobre ellas para poder esclarecer el fundamento fisiopatológico de esta asociación.

\section{AGRADECIMIENTOS}

Los autores expresan su reconocimiento a la Dra. Sara Cano, del Servicio de Medicina Preventiva del Hospital Clínico San Carlos, por el asesoramiento estadístico prestado.

\section{BIBLIOGRAFÍA}

1. Collier J, Sherman M. Screening for hepatocellular carcinoma. Hepatology 1998; 27: 273-8.

2. El Serag HB, Mason AC. Rising incidence of hepatocellular carcinoma in the United States. N Engl J Med 1999; 340: 745-50.

3. Bosch FX, Ribes $\mathbf{J}$, Borràs $\mathrm{J}$. Epidemiology of primary liver cancer. Sem Liv Disease 1999; 19: 271-85.

4. Parkin DM, Bray F, Ferlay J, Pisani P. Estimating the world cancer burden: GLOBOCAN 2000. Int J Cancer 2001; 94: 153-6.

5. Simonetti RG, Cammà C, Fiorello F, Politi F, D’Amico G, Pagliaro L. Hepatocellular carcinoma. A worldwide problem and the major risk factors. Dig Dis Sci 1991; 36: 862-72.

6. Sangro B, Herráiz M, Martínez-González MA, Bilbao I, Herrero I, Beloqui O, et al. Prognosis of hepatocellular carcinoma in relation to treatment: A multivariate analysis of 178 patients from a single European institution [published erratum appears in Surgery 1998; 124: 1087]. Surgery $1998 ; 124: 575-83$.

7. Francazani AL, Conte D, Fraquelli M, Taioli E, Mattioli M, Losco A, et al. Increased cancer risk in a cohort of 230 patients with hereditary hemochromatosis in comparison to matched control patients with non-iron-related chronic liver disease. Hepatology 2001; 33: 647-51.

8. Haddow JE, Palomaki GE, McClain M, Craig W. Hereditary haemochromatosis and hepatocellular carcinoma in males: A strategy for estimating the potential for primary prevention. J Med Screen 2003; 10: 11-3.

9. Elmberg M, Hultcrantz R, Ekbom A, Brandt L, Olsson S, Olsson R, et al. Cancer risk in patients with hereditary hemochromatosis and in their first-degree relatives. Gastroenterology 2003; 125: 1733-41.
10. Bosch FJ, Ribes J. Epidemiology of liver cancer in Europe. Can J Gastroenterol 2000; 14: 621-30.

11. Stickel F, Schuppan D, Hahn EG, Seitz HK. Cocarcinogenic effects of alcohol in hepatocarcinogenesis. Gut 2002; 51: 132-9.

12. Cruz DM, Fernández S, Alba C, Rodríguez-Agulló JL, Ladero JM, Díaz-Rubio M. Doscientos tres casos de hepatocarcinoma: 11 años de experiencia. Rev Esp Enferm Dig 2001; 93 (Supl. 1): 185-6 (Abstract).

13. Feder JN, Gnirke A, Thomas W, Tsuchihashi , Ruddy DA, Basava A, et al. A novel MHC class-I like gene is mutated in patients with hereditary haemochromatosis. Nat Genet 1996; 13: 399-408.

14. Rochette J, Pinton JJ, Fisher CA, Perera G, Arambepola M, Arichchi DS, et al. Multicentric origin of hemochromatosis gene (HFE) mutations. Am, J Human Genet 1999; 64: 1056-62.

15. Carella M, d'Ambrosio L, Totaro A, Grifa A, Valentino MA, Piperno $\mathrm{A}$, et al. Mutation analysis of the HLA-H gene in Italian hemochromatosis patients. Am J Hum Genet 1997; 60: 828-32.

16. Olynyk JK, Cullen DJ, Aquila S, Rossi E, Summerville L, Powell LW. A population-based study of the clinical expression of the hemochromatosis gene. N Engl J Med 1999; 341: 718-24.

17. Bacon BR, Olynyk JK, Brunt EM, Britton RS, Wolff RK. HFE genotype in patients with hemochromatosis and other liver diseases. Ann Intern Med 1999; 130: 953-62.

18. George DK, Powell LW, Losowsky MS. The haemochromatosis gene: a co-factor for chronic liver diseases? J Gastroenterol Hepatol 1999; 14: 745-9.

19. Thorburn D, Curry G, Spooner R, Spence E, Oien K, Halls D, et al. The role of iron and haemochromatosis gene mutations in the progression of liver disease in chronic hepatitis C. Gut 2002; 50: 248-52.

20. Gehrke SG, Stremmel W, Mathes I, Riedel HD, Bents K, Kallinowski B. Hemochromatosis and transferrin receptor gene polymorphism in chronic hepatitis C: impact on iron status, liver injury and HCV genotype. J Mol Med 2003; 81: 780-7.

21. Erhardt A, Maschner-Olberg A, Mellenthin C, Kappert G, Adams O, Donner A, et al. HFE mutations and chronic hepatitis C: H63D and C282Y heterozygosity are independent risk factors for liver fibrosis and cirrhosis. J Hepatol 2003; 38: 335-42.

22. Ladero JM, Ropero P, Ortega L, Taxonera C, González FA, LópezAlonso G, et al. Mutaciones del gen HFE, contenido hepático de hierro y gravedad histológica en la hepatitis crónica por el virus de la hepatitis C. Rev Esp Enferm Dig 2003; 95: 829-32.

23. Ropero P, Villegas A, Fernández M, Agúndez JAG, González FA, Benítez J, et al. C282Y and H63D mutations of the HFE gene in patients with advanced alcoholic liver disease. Rev Esp Enferm Dig 2001; 93: 160-3.

24. Campos J, González A, Loidi L, Barros F, Vieites B, Pérez E, et al. Mutaciones en el gen HFE (C282Y, H63D, S65C) en pacientes alcohólicos con datos de sobrecarga de hierro. Rev Clin Esp 2002; 202: 534-9.

25. Egger NG, Goeger DE, Payne DA, Miskovsky EP, Weinman SA, Anderson KE. Porphyria cutanea tarda: multiplicity of risk factors including HFE mutations, hepatitis $\mathrm{C}$, and inherited uroporphyrinogen decarboxylase deficiency. Dig Dis Sci 2002; 47: 419-26.

26. Bonkovsky HL, Jawaid Q, Tortorelli K, LeClair P, Cobb J, Lambrecht RW, et al. Non-alcoholic steatohepatitis and iron: Increased prevalence of mutations of the HFE gene in non-alcoholic steatohepatitis. J Hepatol 1999; 31: 421-9.

27. Deguti MM, Sipahi AM, Gayotto LC, Palacios SA, Bittencourt PL, Goldberg AC, et al. Lack of evidence for the pathogenic role of iron and HFE gene mutations in Brazilian patients with nonalcoholic steatohepatitis. Braz J Med Biol Res 2003; 36: 739-45.

28. Bugianesi E, Manzini P, D’Antico S, Vanni E, Longo F, Leone N, et al. Relative contribution of iron burden, HFE mutations, and insulin resistance to fibrosis in nonalcoholic fatty lier. Hepatology 2004; 39: 179-87.

29. Niemelä O, Parkkila S, Britton vRS, Brunt E, Janney C, Bacon B. Hepatic lipid peroxidation in hereditary hemochromatosis and alcoholic liver injury. J Lab Clin Med 1999; 133: 451-60.

30. Chenoufi N, Loreal O, Drenou B, Cariou S, Hubert N, Leroyer P, et al. Iron may induce both DNA synthesis and repair in rat hepatocytes stimulated by EGF/pyruvate. J Hepatol 1997; 26: 650-8.

31. Vautier G, Bomford AB, Portman BC, Metivier E, Williams R, Ryder SD. p53 mutations in British patients with hepatocellular carcinoma: clustering in genetic hemochromatosis. Gastroenterology 1999; 117: 154-60. 
32. Marrogi AJ, Khan MA, van Gijssel HE, Welsh JA, Rahim H, Demetris AJ, et al. Oxidative stress and p53 mutations in the carcinogenesis of iron-associated hepatocellular carcinoma. J Natl Cancer Inst 2001; 93: $1652-5$.

33. Kowdley KV. Iron, hemocromatosis, and hepatocellular carcinoma. Gastroenterology 2004; 127: S79-S86.

34. Bonkovsky HL, Naishadham D, Lambrecht RW, Chung RT, Hoefs JC, Nash SR, et al. Roles of iron and HFE mutations on severity and response to therapy during retreatment of advanced chronic hepatitis C. Gastroenterology 2006; 131: 1440-51.

35. Rosmorduc O, Poupon R, Nion I, Wendum D, Feder J, Bereziat C, et al. Differential HFE allele expression in hemochromatosis heterozygotes. Gastroenterology 2000; 119: 1075-86.

36. Brandhagen DJ, Fairbanks VF, Baldus WP, Smith CI, Kruckeberg $\mathrm{KE}$, Schaid DJ, et al. Prevalence and clinical significance of HFE gene mutations in patients with iron overload. Am J Gastroenterol 2000: 95: 2910-4

37. Gochee PA, Powell LW, Cullen DJ, du Sart D, Rossi E, Olynyk JK. A population based study of the biochemical and clinical expression of the H63D hemochromatosis mutation. Gastroenterology 2002; 122: 646-51.

38. Beutler E, Felitti V, Gelbart T, Ho N. The effect of HFE genotypes on measurements of iron overload in patients attending a health appraisal clinic. Ann Intern Med 2000; 133: 329-37.

39. Cogswell ME, Gallagher ML, Steinberg KK, Caudill SP, Looker AC, Bowman BA, et al. HFE genotype and transferrin saturation in the United States. Genet Med 2003; 5: 304-10.

40. Njajou OT, Houwing Duistermaat JJ, Osborne RH, Vaessen N, Vergeer J, Heeringa J, et al. A population based study of the effect of the HFE C282Y and H63D mutations on iron metabolism. Eur J Hem Genet 2003; 11: 225-31.

41. Merryweather-Clarke AT, Worwood M, Parkinson L, Mattock C, Pointon JJ, Shearman JD, et al. The effect of HFE mutations on serum ferritin and transferrin saturation in the Jersey population. Br J Hematol 1998; 101: 369-73.

42. Aguilar-Martínez P, Bismuth M, Picot MC, Thelcide C, Pageaux GP, Blanc F, et al. Variable phenotypic presentation of iron overload in H63D homozygotes: are genetic modifiers the cause? Gut 2001; 48 : 836-42.

43. Moodie SJ, Ang L, Stenner JM, Finlayson C, Khotari A, Levin GE, et al. Testing for haemochromatosis in a liver clinic population: Relationship between ethnic origin, HFE gene mutations, liver histology and serum iron markers. Eur J Gastroenterol Hepatol 2002; 14: 217 21.

44. De Gobbi M, Daraio F, Oberkanins C, Moritz A, Kury F, Fiorelli G, et al. Analysis of HFE and TFR2 mutations in selected blood donors with biochemical parameters of iron overload. Haematologica 2003; 88: 396-401

45. Raddatz D, Legler T, Lynen R, Addicks N, Ramadori G. HFE genotype and parameters of iron metabolism in German first-time blood donors - evidence for an increased transferrin saturation in $\mathrm{C} 282 \mathrm{Y}$ heterozygotes. Z Gastroenterol 2003; 41: 1069-76.

46. Tomatsu S, Orii KO, Fleming SE, Holden CC, Waheed A, Britton RS, et al. Contribution of the H63D mutation in HFE to murine hereditary hemochromatosis. Proc Natl Acad Sci USA 2003; 100: 15788-93.

47. Lebron JA, Bennett MJ, Vaughn DE, Chirino AJ, Snow PM, Mintier GA, et al. Crystal structure of the hemochromatosis protein HFE and characterization of its interaction with transferrin receptor. Cell 1998; 93: 111-23.

48. de Almeida SF, Carvalho IF, Cardoso CS, Cordeiro JV, Azevedo JE, Neefjes J, et al. HFE cross-talks with the MHC class I antigen presentation pathway. Blood 2005; 106: 971-7.

49. Sambrook J, Frisch E, Maniatis T. Molecular cloning: A laboratory manual. Cold Spring Harbor Press. Cold Spring Harbor; 1989.

50. Aguilar P, Jeanjean PH, Masmejean C, Guillard C, Biron C, Rabesan- dratana $\mathrm{H}$, et al. Simple and rapid detection of the newly described mutations in the HLA-H gene. Blood 1997; 89: 1835-6.

51. Sánchez M, Bruguera M, Bosch J, Rodés J, Ballesta F, Oliva O. Prevalence of the Cys282Tyr and His63Asp HFE gene mutations in Spanish patients with hereditary hemochromatosis and in controls. J Hepatol 1998; 29: 725-8.

52. Briceño O, Ropero P, González FA, Polo M, Mateo M, Benavente C, et al. Prevalence of the $\mathrm{C} 282 \mathrm{Y}$ and H63D gene mutations in umbilical cord blood in Madrid (Spain). Hematol J 2003; 4 (Supl. 2): 63 (Abstract).

53. Mariani R, Salvioni A, Corengia C, Esba N, Lanfazame C, De Micheli $\mathrm{V}$, et al. Prevalence of HFE mutations in upper Northern Italy: Study of 1132 unrelated blood donors. Dig Liver Dis 2003; 35: 47981.

54. Chapoutot C, Esslimani M, Joomaye Z, Ramos J, Perney P, Laurent $\mathrm{C}$, et al. Liver iron excess in patients with hepatocellular carcinoma developed on viral C cirrhosis. Gut 2000; 46: 711-4.

55. Blanc J-F, De Ledinghen V, Bernard P-H, de Verneuil H, Winnock M, Le Bail B, et al. Increased incidence of HFE C282Y mutations in patients with iron overload and hepatocellular carcinoma developed in non-cirrhotic liver. J Hepatol 2000; 32: 805-11.

56. Lauret E, Rodríguez M, González S, Linares A, López-Vázquez A, Martínez-Borra J, et al. HFE gene mutations in alcoholic and virus-related cirrhotic patients with hepatocellular carcinoma. Am J Gastroenterol 2002; 97: 1016-21.

57. Hellerbrand C, Pöppl A, Hartmann A, Schölmerich J, Lock G. HFE C282Y heterozygosity in hepatocellular carcinoma: evidence for increased prevalence. Clin Gastroenterol Hepatol 2003; 1: 279-84.

58. Fargion S, Stazi MA, Francazani AL, Mattioli M, Sampietro M, Tavazzi $\mathrm{D}$, et al. Mutations in the HFE gene and their interaction with exogenous risk factors in hepatocellular carcinoma. Blood Cells Mol Dis 2001; 27: 505-11.

59. Francanzani AL, Fargion S, Stazi MA, Valenti L, Amoroso P, Cariani $\mathrm{E}$, et al. Association between heterozygosity for HFE gene mutations and hepatitis viruses in hepatocellular carcinoma. Blood Cell Mol Dis 2005; 35: 27-32.

60. Shi WJ, Chen H, Zhou B, Cheng J. Association of mutations of HFE gene and hepatocellular carcinoma following chronic hepatitis B. Zhonghua Gan Zang Bing Za Zhi 2005; 13: 682-4 (article in chinese).

61. Cauza E, Peck-Radosavljevic M, Ulrich-Pur H, Datz C, Gschwantler M, Schöniger-Hekele M, et al. Mutations of the HFE gene in patients with hepatocellular carcinoma. Am J Gastroenterol 2003; 98: 442-7.

62. Willis G, Bardsley V, Fellows IW, Lonsdale R, Wimperis JZ, Jennings BA. Hepatocellular carcinoma and the penetrance of HFE C282Y mutations: A cross-sectional study. BMC Gastroenterol 2005; 5: 17.

63. Racchi O, Mangerini R, Rapezzi D, Gaetani GF, Nobile MT, Picciotto A, et al. Mutations of the HFE gene and the risk of hepatocellular carcinoma. Blood Cells Mol Dis 1999; 25: 350-3.

64. Pirisi M, Toniutto P, Uzzau A, Fabris C, Avellini C, Scott C, et al. Carriage of HFE mutations and outcome of surgical resection for hepatocellular carcinoma in cirrhotic patients. Cancer 2000; 15: 297-302.

65. Boige V, Castera L, de Roux N, Ganne Carrie N, Ducot B, Pelletier G, et al. Lack of association between HFE gene mutations and hepatocellular carcinoma in patients with cirrhosis. Gut 2003; 52: 1178-81.

66. Beckman LE, Hägerstrand I, Stenling R, Van Landeghem GF, Beckman L. Interaction between haemochromatosis and transferrin receptor genes in hepatocellular carcinoma. Oncology 2000; 59: 317-22.

67. González Hevilla M, de Salamanca RE, Morales P, Martínez Laso J, Fontanellas A, Castro MJ, et al. Human leukocyte antigen haplotypes and HFE mutations in Spanish hereditary hemochromatosis and sporadic porphyria cutanea tarda. J Gastroenterol Hepatol 2005; 20: 456-62.

68. Geier A, Reugels M, Weiskirchen R, Wasmuth HE, Dietrich CG, Siewert E, et al. Common heterozygous hemochromatosis gene mutations are risk factors for inflammation and fibrosis in chronic hepatitis C. Liver Int 2004; 24: 285-94. 